\title{
POUR UNE APPROCHE EXPÉRIMENTALE DE LA RÉCEPTION : ATTITUDES DOCUMENTAIRE ET FICTIONNELLE
}

\author{
Pierre Fastrez ${ }^{1}$
}

\section{Introduction}

Dans le champ des études en communication iconique, le couple conceptuel "documentaire-fiction" a déjà fait couler beaucoup d'encre. Domaine de préoccupation des filmologues, il s'est vu interrogé par la suite par les narratologues et les sémiopragmaticiens, pour ne citer qu'eux. Ce domaine de recherche compte de multiples facettes, articulées autour d'une (double) question centrale : 'qu'estce qu'un film/une image documentaire ?, qu'est-ce qu'un film/une image de fiction?" Cette question regroupe en fait deux champs d'investigations, selon que l'on se place du point de vue du texte ou du point de vue du spectateur ${ }^{2}$. Ces deux grands débats (études du texte et études en réception) sont naturellement intimement liés, et en particulier, les questions tenant de la réception du documentaire et de la fiction ne peuvent que reposer sur une définition préalable des deux objets.

1 Aspirant au Fonds National de la Recherche Scientifique (FNRS) - Belgique.

2 ... ce à quoi on pourrait ajouter logiquement un troisième point de vue, celui du cinéaste, de l'auteur, sujet moins souvent exploité en recherche en communication. 
Les lignes qui suivent tendent à rendre compte d'une étude comparative visant à différencier expérimentalement l'attitude de réception du documentaire de celle de la fiction dans le chef du spectateur. Le but était donc de partir de ce que ce dernier ressent (ou plutôt déclare ressentir), de l'empirie, afin de confronter celle-ci à la modélisation théorique des deux attitudes de réception préexistant dans la littérature, en tentant de dégager l'apport spécifique d'une étude expérimentale face à un tel objet de recherche.

Ayant résolu d'aborder cette problématique du point de vue de la réception, je partirai des définitions des textes documentaires et fictionnels existantes. Deux conceptions s'opposent sur ce sujet. La première repose sur la nature du référent : le documentaire donne à voir des objets existant ailleurs indépendamment de lui, la fiction ne montre que des objets fictifs, et donc inexistants. La seconde s'appuie sur le mode d'énonciation du message :

La distinction se situe au niveau du discours: ce qu'on pourrait dire de la façon suivante : ce qui différencie le documentaire de la fiction, ce n'est pas la nature des objets rapportés mais la nature de l'acte de s'y rapporter, fictif dans un cas, réel dans l'autre'.

Le documentaire serait ainsi le fait d'un énonciateur réel, qui s'adresse au spectateur en tant que personne réelle, par opposition à la fiction, énoncée par un énonciateur fictif", qui "accomplit l'acte d'énoncer «sans assumer les engagements qui sont normalement requis par ces actes» (...) mais aussi «sans intention de tromper» ${ }^{3}$ ('énonciateur fictivisant n'est pas un menteur)"4.

Dans le cadre de cette étude, j'ai décidé de ne pas trancher en faveur de l'une ou l'autre forme de définition, considérant le documentaire comme un message à énonciateur réel et présentant des

1 G. JACQUINOT, "Le Documentaire, une fiction (pas) comme les autres", in Cinémas, revue d'études cinématographiques, vol. $4, \mathrm{n}^{\circ} 2$, Montréal, Québec, Université de Montréal, hiver 1994, pp. 64-65.

2 Roger Odin parle de "je-origine fictive", qu'il oppose au "je-origine réelle" propre au documentaire (Cf. R. ODIN, "Film documentaire, lecture documentarisante", in Cinémas et Réalités, Travaux XLI, R. OdIN et J.-Cl. LyANT (dir.), CIEREC, Université de Saint-Etienne, 1984, pp. 262-280).

3 J. SEARLE, "Le statut logique du discours de la fiction", in Sens et expression, Paris, Éd. du Seuil, 1982, cité dans : R. ODIN, "Du spectateur fictionnalisant au nouveau spectateur, approche sémio-pragmatique", IRIS, $\mathrm{n}^{\circ} 8$ : "Cinéma et narration 2", Paris, 1988, p. 128.

4 R. ODIN, "Du spectateur fictionnalisant...", op. cit., p. 128. 
objets réels existant par ailleurs, et la fiction comme un message à énonciateur fictif présentant des objets inexistants par ailleurs. En effet, le champ des documentaires et des fictions produits est suffisamment hétérogène (et présente suffisamment de cas hybrides) pour qu'on le considère non comme un ensemble ne comportant que deux modalités exclusives (le documentaire pur d'un côté, la fiction pure de l'autre), mais comme un continuum dont les deux pôles (les deux cas les plus typiques) seraient les deux possibilités que je viens de définir.

Les attitudes documentaire et fictionnelle différenciées ici peuvent donc être envisagées comme comportant deux niveaux de lecture, correspondant aux deux critères discriminants exposés plus haut (référent et mode d'énonciation), chaque attitude pouvant s'engager dans le chef du spectateur sur base de l'un comme de l'autre'.

\section{Hypothèses de travail et interprétation théorique}

Ma recherche aura été guidée par quatre hypothèses ${ }^{2}$, qu'il convient d'emblée de détailler. Celles-ci ont pour objet de différencier attitude documentaire et attitude fictionnelle sur base de réponses fournies par des individus interrogés à propos de photographies (documentaires ou fictionnelles) qui leurs sont présentées ${ }^{3}$. Je les exposerai en corrélation avec les thèses théoriques qui les soutiennent.

\section{Hypothèses 1 et 2}

H1. Les spectateurs d'images de fiction ont tendance à être doublement plus loquaces que leurs homologues "documentaires"'4

1 Mon approche expérimentale ne porte presque exclusivement que sur la perception du référent (premier niveau de lecture de l'attitude), sans aborder de front la question de l'énonciation. Cependant, l'analyse détaillée du seul niveau de la référence permet déjà de tirer des conclusions (bien que de façon partielle) sur la manière dont l'énonciateur est perçu.

2 Formulées suite à un prétest mené sur trois personnes. Cf. infra.

3 Cf. infra (descriptif des dispositifs expérimentaux mis en place dans le cadre de cette recherche).

4 Dans la suite de l'exposé, j'utiliserai par facilité les termes "spectateur documentaire" et "spectateur fictionnel" respectivement pour "spectateur d'images documentaires" et "spectateur d'images de fiction". 
- d'une part, d'un point de vue quantitatif (ils répondent plus abondamment à mes questions $)^{1}$;

- d'autre part, du point de vue de la nature de leurs éléments de réponse (leurs réponses comportent plus d'éléments invisibles sur les photographies).

H2. Les spectateurs d'images documentaires font preuve de plus de prudence dans leurs réponses, qu'ils mettent plus facilement en doute que leurs homologues "fictionnels".

D'un point de vue théorique, ces deux hypothèses trouvent des éléments de justification communs. Dans le cas du documentaire, le mutisme et la prudence du spectateur peuvent s'expliquer en partant de la position d'existence $e^{2}$ propre à l'objet représenté. Le spectateur du documentaire pose d'emblée l'objet qu'il observe à l'écran ${ }^{3}$ comme absent -c'est là le caractère d'irréalité propre à l'image ${ }^{4}$ mais comme existant ailleurs. A travers l'objet observé à l'écran, c'est "l’objet-en-général" 5 l'objet réel qui est visé par le spectateur. Dans le cas particulier du personnage présenté à l'écran, la position d'existence de celui-ci rappelle le spectateur à son propre point de vue ${ }^{6}$, et le pousse donc à effectuer, ne fût-ce qu'en partie, le "dédoublement

1 Notons d'emblée que la première partie de cette hypothèse a été rapidement abandonnée, essentiellement pour des raisons pratiques (absence d'indicateurs fiables pouvant la traduire en données traitables).

2 Cf. J.-P. MEUNIER, Les structures de l'expérience filmique: l'identification filmique, Leuven, Librairie universitaire, 1969, p. 65.

3 Dans la suite de l'interprétation théorique, je prendrai pour référence -par facilitéle documentaire et la fiction filmiques. Les considérations qui suivront valent bien entendu aussi pour les autres formes de documentaire ou de fiction.

4 L'image se pose comme substitut du réel, qui se trouve irréalisé par elle. L'objet représenté par l'image est d'emblée posé comme absent par celle-ci. L'idée de substitution doit ici être entendue au sens fort: "Elle concerne moins les choses elles-mêmes que l'expérience que nous en faisons. Les images se posent sur le monde pour faire écran à notre expérience habituelle de celui-ci, ou si l'on veut, pour transformer notre rapport au monde" (J.-P. MEUNIER et D. PERAYA, Introduction aux théories de la communication, Bruxelles, De Boeck-Wesmael, 1993, p. 141).

5 L'expression est, elle aussi, empruntée à J.-P. Meunier (Les structures..., op. cit.).

6 C'est-à-dire qu'elle l'écarte de la double indifférenciation (entre image et réel extérieur, entre lui-même et monde de l'image) propre à la réception iconique, et le pousse à opérer un dédoublement entre les conduites telles qu'il les perçoit, les "revit", et ces mêmes conduites telles qu'elles se déroulent réellement, indépendamment de lui. 
réflexif'" que l'état spectatoriel à tendance à réprimer ${ }^{2}$. Ainsi, l'identification au personnage reste une simple "attitude de compréhension"3. Le spectateur distingue donc l'objet tel qu'il le perçoit de l'objet tel qu'il est réellement ailleurs, les conduites telles qu'il les appréhende des conduites telles qu'elles sont vécues en réalité par le personnage, etc. Ceci, en toute logique, doit l'empêcher de se permettre d'extrapoler, d'inférer à l'objet des traits qu'il ne peut observer. D'où son relatif mutisme, et sa prudence lorsqu'il parle de ce qu'il voit à l'écran ou sur photographie.

Pour le spectateur de fiction, les choses sont bien différentes. Tout d'abord, s'il pose l'objet représenté comme absent, il ne le considère ni comme existant, ni comme inexistant par ailleurs, mais il y croit. Il y a en effet -dans le chef du spectateur de fiction"croyance", et non "position d'existence". Le mode de croyance au monde représenté, propre à la fiction, peut être résumé par la (célèbre) formule d'O. Mannoni : "je sais bien ... mais quand même..."4. Face à la fiction, le spectateur se change en un être au Moi clivé, une part de lui-même étant consciente de la nature fictive de ce qu'il voit ("je sais bien que tout ceci n'existe pas vraiment"), laissant l'autre s'adonner pleinement au jeu de la fiction, et y croire totalement ("... mais quand même j'y crois comme si c'était vrai") ${ }^{5}$. Créant un monde détaché du

1 Le terme est de COHEN-SEAT (Problèmes du cinéma et de l'information visuelle, Paris, Presses Universitaires de France, 1961), repris dans J.-P. MeuniER et D. PERAya, op. cit., p. 138.

${ }^{2}$ Là où, dans l'interaction réelle, nous n'avons jamais accès qu'à des facettes des objets et personnes que nous rencontrons, sans jamais les percevoir dans leur totalité, l'expérience iconique crée une adéquation totale entre nos perceptions et les objets perçus, inexistants en dehors de celles-ci. Les objets que nous percevons ne transcendent donc plus la perception que nous avons d'eux. Le regard d'autrui nous rappelant à notre propre individualité, à la position spatio-temporelle que nous occupons par rapport à lui, est absent dans la perception iconique. Plus rien ne nous pousse à effectuer ce "dédoublement réflexif", corrélatif des conduites d'identification dans l'interaction réelle. (Cf. J.-P. MEUNIER et D. PERAYA, op. cit., pp. 137 à 143, synthétisant l'approche des filmologues des années 50-60 des phénomènes d'identification filmique).

3 Cf. J.-P. MeUniER, Les structures..., op. cit., p. 107.

4 O. MANNONI, Clefs pour l'imaginaire ou l'autre scène, Paris, Éd. du Seuil, 1969.

5 Fondamentalement, c'est parce qu'il sait qu'il assiste à une fiction qu'il peut se laisser berner, l'un étant la condition sine qua non de l'autre. Selon Roger Odin, le mode de croyance propre à la fiction est rendu possible par l'opération de fictivisation, qui pose la fiction comme étant le fait d'un " je-origine fictive ", énonciateur n'assumant pas la véracité de ses propos (Cf. supra (définition de la fiction sur base du mode d'énonciation) et R. ODIN, "Du spectateur fictionnalisant...", op. cit.). 
réel, l'image de fiction pousse la position de réception propre à l'image à son paroxysme : elle met le spectateur dans un état d'indifférenciation (entre lui et le monde de l'image, entre image et réel extérieur), dans lequel il est fortement centré à la fois sur son point de vue et sur les conduites qu'il perçoit ${ }^{1}$.

Se plaçant du point de vue du texte, Edward Branigan parle de "référence partiellement déterminée"2, dans la mesure où l'image fictionnelle ne désigne pas de manière spécifique quel objet elle représente, mais plutôt quelle catégorie d'objets, dont chaque membre peut être référent de l'image ${ }^{3}$. Il incombe donc au spectateur de compléter la référence sur base de son propre vécu, de son expérience du monde (ce qui explique en partie le pouvoir d'interpellation de la fiction ${ }^{4}$ ). L'ensemble de ces éléments rendent le spectateur de fiction totalement libre d'attribuer à l'objet représenté les traits qu'il souhaite, dans la mesure où celui-ci est ce qu'il y projette. Ceci permet d'expliquer sa loquacité et sa moindre prudence comparativement à son homologue documentaire.

\section{Hypothèse 3}

H3. Quand il s'agit de contextualiser la situation présentée par la photographie, les spectateurs d'images fictionnelles ont tendance à réintégrer celle-ci au sein d'un récit, là où leurs homologues "documentaires" ont tendance à la replacer dans une situation présente plus large.

Pour expliquer la collusion entre attitude fictionnelle et "mise en narration", sans doute faut-il chercher du côté de la proximité culturelle entre film de fiction et récit. Tout spectateur occidental moyen s'attend à se voir raconter une histoire quand on lui présente une fiction. De plus, n'ayant pas de référent réel externe, les conduites

1 Ainsi l'état spectatoriel a-t-il été souvent rapproché du rêve par les filmologues des années 50-60, ou plutôt de la rêverie, activité de veille (Pour une comparaison approfondie entre rêve, rêverie et état spectatoriel, on se reportera à l'article de C. METZ, "Le film de fiction et son spectateur (étude métapsychologique)", in Communications, $\mathrm{n}^{\circ} 23$, Paris, Éd. du Seuil, 1975, pp. 108-135).

2 Le concept de "partially determined reference " est développé par Ed. BRANIGAN dans Narrative comprehension and film, Londres, Routledge, 1992, p. 192-217.

3 Branigan prend l'exemple d'une photo fictionnelle d'un tigre pouvant référer à n'importe quel tigre, passé, présent ou à venir.

${ }^{4}$ Le spectateur y projetant plus ou moins ce qu'il veut, il se sent d'autant plus touché par ce qu'il voit. 
perçues à l'écran par le spectateur de fiction sont appréhendées dans leur singularité, pour la signification qu'elles prennent en regard des événements, de la situation fictive (et non plus comme une médiation vers un "objet-en-général"). L'activité de réception peut ainsi être ramenée à un double mouvement, combinant conduites de rétention et de protention ${ }^{1}$. A chaque instant, le spectateur accumule d'une part les informations sur l'objet ou le personnage (rétention), et utilise cellesci d'autre part pour spéculer sur la suite des événements, lancer des hypothèses sur le déroulement ultérieur de l'action, dans le but ultime "de posséder l'objet qu'[il]vise (le film dans son entièreté)"2. L'intégration des conduites observées à une narration apparaît donc comme relativement naturelle à l'attitude fictionnelle.

On peut en outre prendre en compte ce qu'Odin nomme la mise en phase, qui constitue une opération d'homogénéisation du film de fiction, "visant à faire fonctionner toutes les instances filmiques comme des adjuvants du narrateur"3, de sorte qu"à tous les grands moments de l'histoire racontée, le travail du film produi[se] une relation film - spectateur (un positionnement affectif du spectateur) homologue aux relations qui se manifestent dans la diégèse"4. Tous les éléments du film (cadrage, montage, plastique de l'image, son, etc.) sont donc mis au service de la fiction, pour mieux captiver le spectateur. Celui-ci, supposant d'emblée la mise en phase de la fiction qu'il voit, il peut relier chacun des éléments observés à la narration fictionnelle.

La proximité entre attitude documentaire et "mise en situation" peut s'expliquer par l'hypothèse faite par le spectateur selon laquelle image et référent réel sont unis par une relation indicielle: les choses se seraient passées de la même manière si la caméra n'avait pas été là pour les filmer". "The image is the referent projected onto the screen"6, synthétise Bill Nichols. Visant l'objet-en-général à travers

1 Sur ces concepts, cf. J.-P. MEUNIER, Les structures..., op. cit.

2 Ibid., p. 91.

3 R. ODIN, "Du spectateur...", op. cit., p. 127.

4 R. ODIN, "Pour une sémio-pragmatique du cinéma", IRIS, vol. $1 \mathrm{n}^{\circ} 1$ : "État de la théorie. Nouveaux objets. Nouvelles méthodes", Paris, ler trimestre 1983, p. 75.

5 Bill Nichols note que dans certains cas, le spectateur est amené à mettre en doute ce postulat (quand l'influence du cinéaste sur les événements profilmiques est manifeste, par exemple). Cependant, cette mise en doute reste typique de l'attitude documentaire. (B. NICHOLS, Representating reality: issues and concepts in documentary, Bloomington, Indiana University Press, 1991, pp. 24 et suiv.)

6 Ibid., p. 25. 
l'objet représenté, le spectateur effectue un travail de rétention: considérant chaque conduite du personnage, chaque profil de l'objet montré à l'écran comme représentatif du référent réel, il accumule au fur et à mesure ces informations qui lui permettent de constituer un savoir général et cumulatif sur l'objet (ou le personnage). Ceci l'autorise à "inférer le tout à partir de la partie", c'est-à-dire à reconstruire une situation présente (synchronique) plus large sur base de ce qui lui est montré. Cependant, dans la mesure où il reste toujours dans un rapport d'extériorité vis-à-vis du personnage ou de la situation qu'il pose comme existant ailleurs (et qui transcende sa perception), il lui est impossible d'inférer ce qu'il ne peut voir, c'est-à-dire en l'espèce un passé et un futur à la situation. Le spectateur est animé d'un "désir de savoir", qui selon B. Nichols, permet d'expliquer le plaisir filmique propre au documentaire :

Knowledge, as much or more than the imaginary identification between viewer and fictional character, promises the viewer a sense of plenitude or self-sufficiency. Knowledge, like the ideal-ego figures or objects of desire suggested by the characters of narrative fiction, becomes a source of pleasure that is far from innocent ${ }^{1}$.

\section{Hypothèse 4}

H4. Dans leurs réponses, les spectateurs d'images documentaires font plus facilement référence à la prise de la photographie, au photographe, à la pose que leurs homologues "fictionnels".

Affirmer de façon catégorique que seuls les spectateurs documentaires peuvent avoir conscience de se trouver devant une photographie (ou une prise de vue) prise à un moment donné serait abusif. Par contre, on peut supposer que si les spectateurs fictionnels comme les documentaires peuvent en être conscients, le mode de croyance qui est propre aux premiers occulte cette connaissance implicite : le spectateur de fiction sait bien que ce n'est qu'une fiction (qui a été tournée à telle date, etc.), mais quand même il y croit et y adhère pleinement, en faisant comme s'il ne le savait pas.

A l'opposé, la relation indicielle image - référent supposée par le spectateur documentaire maintient chez lui la conscience de l'écart entre le réel et la représentation. Et quand bien même cette

1 Ibid., p. 31. 
supposition est mise en doute, c'est pour suspecter l'influence du photographe ou du cinéaste sur le motif profilmique ${ }^{1}$, ce qui ne peut qu'accroître ladite conscience.

\section{Méthodologie}

Ma démarche expérimentale aura suivi un déroulement relativement classique, empruntant les étapes suivantes : pré-test, test et analyse quantitative des données, résultats, post-test sur échantillon réduit et analyse qualitative du post-test, conclusions. C'est suite au pré-test qu'ont été formulées les quatre hypothèses discriminant attitude documentaire et attitude fictionnelle, la suite de mes investigations étant destinée à infirmer ou à confirmer celles-ci.

Les différents tests mis au point dans le cadre de cette recherche ont consisté en un dispositif relativement similaire. Il s'agissait à chaque fois de confronter un individu à une ou plusieurs photographie(s) légendée(s) ${ }^{2}$, pour lui poser ensuite une série de questions concernant celle(s)-ci.

Le pré-test a été mené sur trois personnes. Les trois interrogés se voyaient présenter les trois mêmes photographies, portant chacune une légende à caractère soit documentaire soit fictionnel (la légende différant selon l'interrogé). Ils étaient ensuite priés de répondre par écrit à quatre questions portant sur le personnage et l'environnement spatio-temporel représenté.

Le test proprement dit a été administré à 46 étudiants du secondaire (entre 16 et 20 ans). Il comprenait un questionnaire (identique pour tous) comprenant 18 questions écrites, ainsi qu'une photographie (identique pour tous ${ }^{3}$ ) légendée. La légende était soit fictionnelle ( 21

1 Notons que si la différence se marque de manière probante dans mes expériences (cf. infra), c'est très probablement justement parce que les personnages de la photographie utilisée posent manifestement, ce qui pousse les spectateurs documentaires (et eux seuls) à mettre en doute ladite relation indicielle.

2 L'expérience a dû se limiter à un support photographique pour des raisons essentiellement pratiques: d'une part aucun vidéogramme susceptible d'être considéré aussi bien comme documentaire que comme fiction n'a pu être trouvé (ou réalisé) ; d'autre part, toute réalisation filmique comporte par essence beaucoup plus d'indices permettant de la rattacher à un "genre" (qualité sonore, mouvements de caméra, traces d'énonciation) que n'en comporte une photographie.

3 La photographie retenue pour le test était l'une des trois utilisées pour le prétest. Elle représente un groupe de 7 policiers (dont une dame qui se trouve en être le chef) posant devant un stade. Cette photo a été retenue pour son ambiguité, 
questionnaires) soit documentaire (25 questionnaires). Les étudiants étaient invités à regarder attentivement la photographie et sa légende et à répondre par écrit en fonction de leurs impressions personnelles. Un espace de trois lignes était prévu pour chaque réponse. Les questions ${ }^{1}$ portaient sur la situation et le décor (questions 1 à 3 ), sur les différents protagonistes en présence (questions 4 à 6 ), et sur le personnage principal (questions 7 à 18). Les questions sur ce dernier portaient sur son physique et son attitude (quatre questions), sur ses sentiments présents (deux questions), sur son métier de policier (deux questions), sur sa personnalité (deux questions), sur sa vie quotidienne (une question) et son passé (une question).

Suite à l'analyse des données produites par le test, j'ai été amené à mettre sur pied un post-test, qui m'aida à confirmer certaines de mes hypothèses. Ce post-test a consisté en deux interviews. Les deux interrogés se voyaient présenter la photographie du test, accompagnée d'une légende fictionnelle pour l'un, documentaire pour l'autre, et étaient ensuite invités à répondre oralement à une série de questions similaires à celles du test, en se sachant enregistrés. Cette méthodologie m'a permis de prendre succinctement en compte des paramètres non textuels (intonation, hésitations, gêne, etc.) que le test avait laissé de côté. $\mathrm{Vu}$ le nombre restreint d'interrogés, les résultats nouveaux mis en lumière par ce post-test n'ont pu dépasser le stade des hypothèses encore à éprouver.

\section{Analyse des données et résultats}

Après une première lecture exploratoire des données récoltées ${ }^{2}$, j'ai traduit mes hypothèses en indices mesurables, de façon à pouvoir opérer un relevé systématique sur tous les questionnaires. Lesdits indices sont repris dans le tableau suivant:

empêchant de juger d'emblée s'il s'agissait d'une image documentaire ou fictionnelle.

1 Cf. annexe.

2 C'est-à-dire les réponses aux 46 questionnaires décrits plus haut. 


\begin{tabular}{|l|l|}
\hline \multicolumn{1}{|c|}{ Hypothèse } & \multicolumn{1}{c|}{ Indice(s) } \\
\hline $\begin{array}{l}\text { Fictionnels plus loquaces que } \\
\text { documentaires }\end{array}$ & $\begin{array}{l}\text { - non réponses }(d t) ; \\
\text { - réponses "je ne sais pas" }(d t) ; \\
\text { - nombre de réponses "déviantes" }(d t)\end{array}$ \\
\hline $\begin{array}{l}\text { Fictionnels moins prudents que } \\
\text { documentaires }\end{array}$ & $\begin{array}{l}\text { - marques de prudence }(d t) ; \\
\text { - réponses "je ne sais pas" }(d t) ; \\
\text { - conscience d'inventer }{ }^{2}(d c) ;\end{array}$ \\
\hline $\begin{array}{l}\text { Mise en narration fictionnelle } \\
\text { mise en situation documentaire }\end{array}$ & $\begin{array}{l}\text { - présence d'un lien passé-situation } \\
\text { présente dans la réponse }(d c) ; \\
\text { nombre de réponses "déviantes" } \\
(d t) \text {; }\end{array}$ \\
\hline $\begin{array}{l}\text { Référence à la photographie } \\
\text { documentaire }\end{array}$ & $\begin{array}{l}\text { - présence d'une référence à la } \\
\text { photographie, à la pose, au } \\
\text { photographe, etc. }(d c)\end{array}$ \\
\hline
\end{tabular}

Indices relevés

La mention "réponses déviantes" figurant dans ce tableau mérite une explication. Étant dans l'impossibilité de contrôler dans quelle mesure mes questions pouvaient engendrer des réponses "conformistes" (l'interrogé répondant dans un sens déterminé parce que la question va dans ce sens ${ }^{3}$ ), j'ai dû me limiter à comptabiliser les réponses s'écartant de la réponse-type attendue ${ }^{4}$. Ainsi, pour

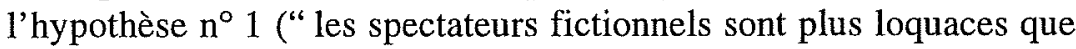
les documentaires "), les réponses déviantes sont celles qui comprennent des éléments invisibles sur la photo quand la question requiert une pure description des objets représentés (réponse dénotant selon mes hypothèses une attitude fictionnelle), ainsi que celles comprenant des éléments descriptifs quand la question porte sur des éléments non observables à l'image (réponse dénotant une attitude documentaire). De même, pour l'hypothèse $\mathrm{n}^{\circ} 3$ (" mise en narration fictionnelle $v s$

1 C'est-à-dire les mentions du type "peut-être (que)...", "on dirait que...", etc.

2 C'est-à-dire la manifestation dans les réponses de l'interrogé du fait qu'il est conscient d'inventer des éléments de réponse sur commande.

3 Par exemple, à une question du type: "Décrivez sommairement le passé du protagoniste de la photographie", on peut imaginer que quelqu'un invente consciemment un passé au personnage qu'il voit parce qu'on le lui demande, que la photographie soit documentaire ou fictionnelle.

4 A la même question, l'interrogé "déviant" répondrait par des éléments présents de la situation présentée sur la photographie. 
mise en situation documentaire), les réponses déviantes correspondent à celles qui se réfèrent au passé quand la question porte sur le contexte présent (réponse "fictionnelle") et vice versa (réponse "documentaire").

Ce "défrichage" des questionnaires "bruts" a engendré une masse de données chiffrées, qui ont pu faire l'objet d'un traitement statistique, ayant pour but la comparaison systématique des deux souséchantillons interrogés (sujets documentaires et fictionnels) ${ }^{1}$. Ce traitement a été mené de façon différente selon que les variables étudiées étaient dichotomiques (présence / absence d'un phénomène, indices marqués " $(d c)$ ") ou discrètes (nombre de mentions de tel type ..., indices marqués " $(d t)$ "). L'analyse de chaque variable a pris pour point de départ l'examen comparé des distributions et des scores moyens des deux sous-échantillons, permettant de diagnostiquer une différence à vérifier par le biais de l'analyse statistique. Pour les variables dichotomiques, donnant des tables $2 \times 2$, le test de divergence de Pearson $\left(\chi^{2}\right)$ a été appliqué, ainsi que le coefficient de corrélation (non paramétrique) qui en découle $\left(\phi=\sqrt{ }\left(\chi^{2} / n\right)\right)$, et le coefficient de corrélation (r) paramétrique de Bravais-Pearson. Pour les variables discrètes, le même test de corrélation de Bravais-Pearson a été employé, couplé avec le test d'égalité des moyennes d'échantillons indépendants de Fisher-Snedecor.

Au cours de l'analyse des données proprement dite, force fut de constater que les tendances postulées dans mes hypothèses n'étaient pas aussi tranchées que ce à quoi on aurait pu s'attendre. Pour un certain nombre d'indices, les deux échantillons (spectateurs documentaires et fictionnels) se comportaient de façon relativement similaire. Au nombre de ces indices, on peut citer les taux de non réponses et les réponses de type "je ne sais pas" (hypothèse $\mathrm{n}^{\circ} 1$ et 2 ), les taux de réponses "déviantes" aux questions descriptives (hypothèse $n^{\circ} 1$ ) et aux questions portant sur le contexte général de la photographie (hypothèse $n^{\circ} 3$ ), ainsi que les réponses manifestant une conscience d'inventer délibérément (hypothèse $\mathrm{n}^{\circ} 2$ ).

On peut -à mon sens- évoquer au moins deux raisons ayant pu empêcher les différences attendues d'émerger. D'une part, il semble

1 Notons que le relevé des occurences des différents indices dans les questionnaires a été mené "en aveugle", c'est-à-dire sans que le " dépouilleur" sache s'il avait affaire à un questionnaire documentaire ou fictionnel. 
que l'on puisse attribuer à la réception de la photographie un "effet documentaire" non attribuable au film. C'est ce que soutiennent André Gaudreault et Philippe Marion quand ils affirment :

(...) comme elle est ontologiquement incapable de reproduire de façon fidèlement analogique le flux temporel, la photographie est généralement perçue comme un "avoir été là authentique" plus que comme une "illusion d'avoir été là". Le film, par contre, est davantage perçu comme une "illusion d'être là" que comme un "être là authentique"l.

La photographie de fiction susciterait le "je sais bien ..." sans laisser le "mais quand même..." s'enclencher2.

D'autre part, la légende fictionnelle du test présentait la photographie comme provenant d'un téléfilm. Le référent de la photographie pouvait donc être compris comme étant le film (objet réel) et non la diégèse fictive à laquelle il renvoyait. La photographie de fiction se mue alors en "photographie documentaire d'un film de fiction", amenant parfois les interrogés à formuler des réponses du type : "je ne sais pas, je n'ai pas vu le film".

Ces deux "biais" dans le dispositif expérimental ont donc pu empêcher les spectateurs fictionnels de se démarquer plus ${ }^{3}$. En effet, des différences plus marquées (dans les mêmes tendances) apparaissent dans les interviews complémentaires, où la légende fictionnelle est celle d'un roman-photo (la photographie réfère donc directement à une diégèse, et non plus à une œuvre réelle existant par ailleurs $)^{4}$.

Néanmoins, mes hypothèses de travail se sont vues confirmées en plusieurs points, qu'il s'agit à présent de détailler. Remarquons d'emblée que dans plusieurs cas, les résultats du test ont mis au jour une relation asymétrique par rapport à la tendance postulée. Ainsi, pour les hypothèses $n^{\circ} 1$ et 3 , seuls les spectateurs documentaires se

1 A. Gaudreault et Ph. Marion, "Dieu est l'auteur des documentaires...", Cinémas, revue d'études cinématographiques, vol. $4 \mathrm{n}^{\circ} 2$, Montréal, Québec, Université de Montréal, hiver 1994, p. 20.

2 Cf. supra.

3 Cf. supra.

4 De plus, cette analyse complémentaire, vu le dispositif mis en œuvre (interrogation orale et enregistrée), a pu porter sur les dimensions "analogiques" des réponses (intonations, embarras manifeste, etc.), et élargir d'autant les voies d'interprétation. Évidemment, les conclusions issues de ces interviews ne sont pas généralisables, dans la mesure où la grille d'analyse des données n'était pas aussi stricte et fiable que lors du test, et où le nombre d'interrogés fut limité à deux. 
démarquent dans le sens attendu, alors que les spectateurs fictionnels manifestent un comportement "neutre".

Ceci est particulièrement représentatif de la conduite des spectateurs fictionnels, qui semblent répondre la plupart du temps "à la demande"1. Ainsi, pour les deux hypothèses précitées, pour lesquelles les indices portaient (entre autres) sur les réponses dites "déviantes"2, en aucun cas ils ne fournissent plus de réponses de ce type que leurs homologues documentaires. Les spectateurs fictionnels répondent aux questions en fonction de ce qu'on leur demande : réponses descriptives aux questions descriptives, réponses inventées aux questions portant sur des éléments non observables, etc.

On peut cependant relever deux points sur lesquels les spectateurs fictionnels se départissent de la "norme". D'une part, ils répondent plus fréquemment (que leurs homologues documentaires) "je ne sais pas" aux questions portant sur le contexte présent de la photographie, ce qui conforte partiellement (et de façon détournée) ma troisième hypothèse. D'autre part, une différence se marque concernant l'indice [lien passé-présent] dans cette même hypothèse $n^{\circ} 3 . \mathrm{Si}$ le nombre de questionnaires où l'interrogé fait explicitement mention d'un lien existant entre le passé du personnage et sa situation actuelle est similaire dans les deux échantillons, on peut en effet noter que seuls les spectateurs fictionnels mentionnent ce lien sans qu' on aie à le leur demander (la dernière question du questionnaire incite à formuler un tel lien). Ils sont donc les seuls à effectuer ce lien de toute façon, là où les spectateurs documentaires ne le font que si on le leur demande.

Comme je le mentionnais plus haut, les résultats ne prennent tout leur relief qu'à l'examen des données de l'échantillon documentaire. Les spectateurs documentaires interrogés se caractérisent, de façon générale, par leur prudence et par leur refus d'inventer des éléments de réponse. Ainsi, les questions auxquelles ils répondent le plus souvent par "je ne sais pas" sont celles portant sur le passé du personnage (auquel ils n'ont par définition pas accès). De même, ils fournissent nettement plus de réponses "déviantes" aux questions portant sur des éléments non visibles (hypothèse $\mathrm{n}^{\circ} 1$ ), ou sur le passé du

1 C'est-à-dire en fournissant le type de réponse que j'ai qualifié plus haut de "conformiste": ils répondent par des éléments du contexte aux questions contextuelles, par des éléments du passé du personnage quand on le leur demande, etc.

2 Cf. supra. 
personnage (hypothèse $\left.n^{\circ} 3\right)^{1}$. Dans les deux cas, ils se réfugient dans ce qu'ils peuvent observer pour ne pas avoir à inventer. Le spectateur documentaire n'infère que sur base des objets ou conduites qu'il observe, et qu'il considère comme représentatifs de la réalité extérieure représentée.

On peut ajouter à cela que (globalement) les spectateurs documentaires fournissent plus de marques de prudence dans leurs réponses que leurs homologues fictionnels (hypothèse $\left.\mathbf{n}^{\circ} 2\right)^{2}$. Cette différence s'avère particulièrement significative dans les questions portant sur des éléments invisibles sur la photographie. Enfin, conformément à ma quatrième hypothèse, les spectateurs documentaires font nettement plus souvent mention de la présence du photographe, de la pose, du fait que la photographie a été prise à un moment donné, etc. ${ }^{3}$.

Tentons de synthétiser brièvement les acquis de cette démarche expérimentale.

Les spectateurs documentaires ont -plus que les fictionnelsconscience que ce qui leur est montré a été photographié à un moment donné. Ils émettent plus de marques de prudence dans leurs affirmations sur l'objet de la photographie, en particulier quand les questions portent sur le passé de l'objet ou du personnage (passé qui ne leur est pas accessible). Ils refusent d'extrapoler ce qu'ils ne peuvent observer dans l'image, et préfèrent ériger les traits et conduites observés en généralités.

On ne peut affirmer, contrairement à l'une de mes hypothèses de travail, que les spectateurs fictionnels infèrent plus (quantitativement)

1 Pour les questions portant sur des éléments invisibles sur la photographie, si l'on croise les variables "type de questionnaire" et "taux de réponses déviantes", on obtient le coefficient de corrélation suivant : (pour les réponses ne mentionnant que des éléments observables) $r=0,312$ ( $\mathrm{r} 40 ; 0,95=0,257$ ). Pour les questions portant sur le passé du personnage, si l'on croise les variables "type de questionnaire " et "taux de réponses déviantes", on obtient le coefficient de corrélation suivant : (pour les réponses ne mentionnant que la situation présente) $r=0,4619$.

2 Le coefficient de corrélation (de Bravais-Pearson) croisant le type de questionnaire avec le taux de marques de prudence par questionnaire donne le résultat suivant : r $=0,2774$.

3 Si l'on croise le type de questionnaire avec la variable "présence/absence d'une référence à la photo", on obtient les résultats suivants : $r=0,487532$ et $\phi=0,48606$ ( $\mathrm{r} 40 ; 0,995=0,393)(\phi=$ test de corrélation non paramétrique); $\chi^{2}=10,8776\left(\chi^{2} 1 ; 0,995=7,88\right)$. 
au sujet de ce qu'ils observent que les spectateurs documentaires. On peut par contre soutenir qu'ils infèrent différemment. Ils n'hésitent pas à imaginer des éléments ou des attributs extérieurs à l'image et indépendants de ce qu'ils peuvent observer, là où leurs homologues documentaires infèrent sur la seule base de ce qu'ils voient. Cette plus grande facilité à extrapoler dans le chef des spectateurs d'images fictionnelles explique qu'ils aient tendance à répondre "à la demande" à mes questions ${ }^{1}$. D' autre part, ces mêmes spectateurs "fictionnels" ont tendance -plus que les spectateurs d'images documentaires- à effectuer un lien entre le passé des personnages fictionnels et la situation présente de l'image, et ce sans qu'il le leur ait été demandé.

\section{Éléments conclusifs}

Deux questions se posent face aux résultats qui viennent d'être détaillés. D'une part, sur base de ces résultats empiriques, que peut-on confirmer (ou infirmer) du modèle théorique que j'ai décrit en début de parcours? D'autre part, dans quel sens de telles conclusions pousseraient-elles à poursuivre la recherche afin de dépasser les limites d'une telle étude ? Envisageons-les dans l'ordre.

Pour les spectateurs documentaires, la confirmation de ma quatrième hypothèse tend à prouver l'existence de la supposition d'une relation indicielle entre image et référent : le spectateur suppose que "les choses se seraient passées de la même façon si le cinéaste n'avait pas été là", et si l'influence de ce dernier est manifeste (comme sur la photographie du test), cette supposition est remise en doute. Par opposition, une telle supposition n'est même pas soulevée par le spectateur fictionnel. Plus fondamentalement, les marques de prudence des spectateurs documentaires et leur refus d'inférer des éléments auxquels ils n'ont pas accès marquent la position d'existence propre à l'attitude documentaire : le personnage représenté par l'image existe ailleurs, transcende la perception (toujours partielle)

1 En effet, le comportement des spectateurs fictionnels, s'il va quelque peu à l'encontre de mes hypothèses, ne contredit pas le modèle théorique décrit plus haut. Leur capacité supérieure à inférer ne leur impose nullement de ne plus tenir compte de ce qu'ils observent. Il semble donc logique qu'ils répondent aux questions descriptives par des descriptions, et aux questions portant sur ce qu'ils ne peuvent voir par des extrapolations. 
que je peux en avoir, je ne peux donc lui inventer une personnalité, un passé, etc.

Pour les spectateurs fictionnels, la "liberté d'invention" dont ils témoignent tend à conforter la conception de l'appréhension des objets fictionnels. Dans un état de double indifférenciation (entre image et référent, entre lui-même et monde le externe), le spectateur n'est plus amené à distinguer l'objet qu'il perçoit de ce qu'il y projette, puisque ledit objet n'existe pas en dehors de sa perception. Le spectateur n'a donc pas le sentiment d' "inventer" ou de "mentir" puisque ce qu'il invente est constitutif de l'objet fictionnel. Pour le dire en d'autres termes, et d'un autre point de vue, le mode de référence "partiellement déterminée" propre à la fiction laisse le spectateur libre de compléter ladite référence comme bon lui semble.

Le lien entre passé et présent du personnage opéré sans qu'on le demande par les spectateurs fictionnels confirme, lui, le jeu dynamique intervenant entre les conduites de rétention et de protention dans l'attitude fictionnelle. En effet, le spectateur de fiction considère les conduites qu'il observe pour la signification qu'elles ont dans l'action fictive, et il relie les premières à la seconde en accumulant d'une part les informations à chaque instant (rétention) et en spéculant sur la suite de l'action sur base de ces informations (protention). Ceci va de pair avec la supposition que tout dans la fiction sert la narration (mise en phase), et donc que tout ce qui est montré prend sens par rapport l'action générale présentée par la fiction.

On le voit, les confirmations que l'on peut tirer du modèle théorique décrit plus haut sur base de ma démarche expérimentale sont relativement restreintes. Tentons, comme annoncé plus haut, d'évaluer les limites de cette dernière, afin d'ouvrir des pistes qui permettront de les dépasser.

Les principales limites de cette recherche résident dans le fait que son approche expérimentale n'aborde pour les deux attitudes que le niveau de lecture propre au référent, et ce d'un point de vue général. Au sein de ce niveau de lecture d'abord, il est une conduite propre à l'état spectatoriel qui n'aura pas été approchée de façon directel $^{1}$ l'identification, " cette conduite posturale par laquelle je

1 ... si ce n'est lors des interviews complémentaires. Lors de ces deux interviews, j'ai demandé aux deux interrogés "comment ils se sentiraient s'ils étaient le personnage dans la situation où il est". Alors que l'interrogé "fictionnel" a joué pleinement le jeu de l'identification, en me faisant part de ses sentiments 
dépasse la simple appréhension des conduites d'autrui pour les revivre de manière active en les incorporant ou, comme disent les psychanalystes, en les «introjectant»"'. Ceci n'a pas empêché que l'analyse des questionnaires "documentaires", par exemple, mette en évidence le rapport d'extériorité qui caractérise la relation spectateur documentaire/personnage. Les modalités de l'identification filmique propres à la fiction ${ }^{2}$ auront par contre été passées sous silence. Il serait à ce titre intéressant de poursuivre la comparaison expérimentale des deux attitudes étudiées à partir des formes d'identification filmique qui leur sont propres, en corrélation avec des notions comme celles de centration ou de décentration ${ }^{3}$.

Ensuite, le niveau de lecture correspondant au mode d'énonciation a été pratiquement ignoré lors de mes expériences. Il constitue donc un domaine d'étude complémentaire à celui traité dans ces lignes. Cette analyse complémentaire est d'autant plus intéressante dans le cadre de la problématique qui nous occupe que ces deux niveaux de lecture ne sont manifestement pas indépendants. En effet, dans quelle mesure peut-on supposer une relation indicielle entre un objet filmique et son référent quand l'énonciation du message dans lequel il apparaît est fictive ? Une telle relation entre image et référent suppose nécessairement d'une part un énonciateur réel, garant de la réalité des objets montrés, et d'autre part l'absence d'influence de celui-ci sur le motif profilmique, comme le mentionnent André Gaudreault et Philippe Marion, qui parlent de "«désolidarisation» du

\footnotetext{
"potentiels" dans une telle situation, l'interrogé "documentaire", lui, a détourné la question, répondant que l'attitude du personnage ne correspondait pas à "son style" à lui. Ces deux réponses viennent conforter les conceptions respectives de l'identification documentaire (identification- compréhension) et de l'identification fictionnelle (participation), mais ne peuvent être considérées comme représentatives, vu le nombre restreint d'interrogés.

1 J.-P. MEUNIER, Les structures ..., op. cit., p. 21.

2 Qualifiée par J.-P. Meunier de participation (dans le cas de l'identification aux personnages fortement " affectivisés" par le spectateur), que celle-ci s'établisse sur le mode de l'être avec (un rapport d'extériorité étant maintenu entre spectateur et personnage, au-delà de la complicité qui les rapproche) ou sur le mode de l'être comme, tentative de fusion entre l'intériorité du spectateur et l'extériorité du personnage, investi pour sa façon d'être au monde, de se comporter. (Cf. J.-P. MEUNIER, Les structures..., op. cit, p. 115 et suiv.)

3 Pour un approfondissement théorique des rapports entre ces notions, cf. notamment J.-P. Meunier et D. PERAYA, op. cit., pp. 234 et suiv., ainsi que J.-P. MEunier, "Image, cognition, centration, décentration", in Cinémas, revue d'études cinématographiques, vol. $4, \mathrm{n}^{\circ} 2$, Montréal, Québec, Université de Montréal, hiver 1994, pp. 27-47.
} 
narrateur (responsable du montage) et du monstrateur (responsable notamment du tournage)"1 dans le documentaire. Les deux instances y fonctionnent de manière distincte :

La monstration documentaire ne consisterait alors non pas en la "production d'un simulacre analogique" mais plutôt, bel et bien, en la simple "transmission d'une analogie" (...). Charge alors au narrateur de relier -de reconfigurer, de lier par son récit- ce matériau profilmique capté - transmis par le monstrateur ${ }^{2}$.

Corrélativement, quand bien même la fiction porte sur un objet réel, l'énonciateur n'assumant pas ses propos, ce qui apparaît à l'image n'est donc plus censé être le reflet de la réalitée, mais jouer un rôle dans l'économie générale du récit, de par l'opération de mise en phase exposée plus haut.

Dans le cadre d'un approfondissement de la question de recherche qui m'a occupé ici, on aurait donc tout intérêt à aborder le niveau de réception de l'énonciation non pas seul, mais de front avec celui du référent, afin de déterminer d'éventuelles contagions entre les deux. Une telle étude pourrait par ailleurs s'intégrer dans une problématique plus large, non abordée ici : celle de la mise en œuvre de lectures spécifiques. Un débat connexe à celui traité ici porte en effet sur les facteurs déterminant le mode de lecture effectivement mis en œuvre face à l'image ou au film. Plusieurs auteurs s'accordent sur le fait qu'en dernière instance c'est le spectateur et lui seul qui détermine le type de lecture adopté, l'image ne contenant pas de consignes contraignant celui-ci. Mais ceci ne fait pas la lumière sur ce en fonction de quoi le spectateur adopte une attitude donnée. Dans la mesure où l'on considère attitude documentaire et attitude fictionnelle non pas comme deux modalités exhaustives et exclusives, mais comme deux positions sur un continuum d'attitudes possibles, la façon dont le spectateur perçoit l'énonciateur du message ou son référent, ainsi que les interactions entre les deux pourraient se révéler déterminantes dans la mise en œuvre d'une attitude particulière.

1 A. Gaudreault et Ph. Marion, op. cit., p. 22.

Ibid.

3 Ainsi, quand un spectateur considère d'un point de vue documentaire les décors d'un film de fiction, il est contraint de construire l'image d'un énonciateur réel (correspondant, par exemple, à la caméra) assumant ses propos, distinct de l'instance d'énonciation du récit fictionnel. 


\section{Annexe : questionnaire du test}

Chaque question était suivie d'un espace de trois lignes pointillées prévu pour la réponse (à l'exception de la dernière question, dont l'espace était de 5 lignes).

[ 1] Décrivez la situation.

[ 2] Quelle est l'atmosphère de la situation ?

[ 3] Décrivez sommairement le décor.

[ 4] Qualifiez (en quelques mots) les différents protagonistes en présence.

[ 5] Qu'est-ce qui distingue la dame du centre des autres protagonistes?

[ 6] Quels rapports entretiennent-ils ?

[ 7] Décrivez physiquement la dame du centre (E. McMurray).

[ 8] Quelle attitude a-t-elle ?

[ 9] Quelle impression dégage-t-elle?

[10] Quels sont les éléments qui suscitent cette impression?

[11] Quels sentiments éprouve-t-elle à ce moment ?

[12] Pourquoi éprouve-t-elle de tels sentiments ?

[13] Pourquoi est-elle entrée dans la police ?

[14] Décrivez le flic McMurray (son caractère, ses habitudes, etc.)

[15] Décrivez la femme McMurray, les traits marquants de son caractère dans la vie.

[16] D'où lui viennent ces traits ?

[17] Décrivez sommairement sa vie de tous les jours.

[18] Décrivez sommairement son passé. 